\title{
Radiolabeled Fucoidan as a P-Selectin Targeting Agent for In Vivo Imaging of Platelet-Rich Thrombus and Endothelial Activation
}

\author{
François Rouzet ${ }^{* 1-3}$, Laure Bachelet-Violette ${ }^{* 1,4}$, Jean-Marc Alsac ${ }^{1}$, Michimasa Suzuki ${ }^{1}$, Alain Meulemans ${ }^{1-3}$, \\ Liliane Louedec ${ }^{1}$, Anne Petiet ${ }^{5}$, Martine Jandrot-Perrus ${ }^{1}$, Frédéric Chaubet ${ }^{1,4}$, Jean-Baptiste Michel ${ }^{1}$, \\ Dominique Le Guludec ${ }^{1-3}$, and Didier Letourneur ${ }^{1,4}$ \\ ${ }^{1}$ INSERM, U698, Cardiovascular Bioengineering, Paris, France; ${ }^{2}$ Univ. Paris Diderot, Sorbonne Paris Cité, Paris, France; \\ ${ }^{3}$ Department of Nuclear Medicine, Bichat-Claude Bernard Hospital, AP-HP, Paris, France; ${ }^{4}$ Institut Galilée, University Paris 13, \\ Villetaneuse, France; and ${ }^{5}$ Institut Claude Bernard-ICB 2, UFR de Médecine, Bichat, France
}

\begin{abstract}
P-selectin expression is involved in the pathophysiology of biologically active arterial thrombus and endothelial activation after a transient ischemic event. Fucoidan is a polysaccharidic ligand of P-selectin, with a nanomolar affinity. In the present study, we propose a new approach of P-selectin molecular imaging based on radiolabeled fucoidan. Methods: Two kinds of experimental models were selected to evaluate the ability of radiolabeled fucoidan to detect $\mathrm{P}$-selectin expression: plateletrich arterial thrombi (vegetations of infective endocarditis and arterial mural thrombus) and myocardial ischemia-reperfusion. These 2 settings were chosen because they were clinically relevant, and both were associated with an important overexpression of platelet and endothelial P-selectin, respectively. Results: ${ }^{99 m T c-f u c o i d a n ~ S P E C T ~ w a s ~ a b l e ~ t o ~ d e t e c t ~ t h e ~ p r e s-~}$ ence of platelet-rich arterial thrombi in all animals, with a median target-to-background ratio of 5.2 in vegetations of endocarditis and 3.6 in mural aneurysmal thrombus, and to detect a persistent endothelial activation at $2 \mathrm{~h}$ after reperfusion. In this latter model, the magnitude of the signal was correlated with the extent of myocardium that underwent transient ischemia. The sensitivity of selectivity of the uptake and retention of $99 \mathrm{mTc}$-fucoidan in both settings was excellent. Conclusion: This study supports $99 \mathrm{~m} T \mathrm{c}$-fucoidan as a relevant imaging agent for in vivo detection of biologic activities associated with P-selectin overexpression, such as arterial thrombus and ischemic memory. Given the reported wide availability at a low cost, and its low toxicity, fucoidan seems to overcome some of the limitations of previous P-selectin-targeted imaging agents.
\end{abstract}

Key Words: P-selectin; fucoidan; thrombus; ischemia; imaging

J Nucl Med 2011; 52:1433-1440

DOI: 10.2967/jnumed.110.085852

\footnotetext{
Received Nov. 24, 2010; revision accepted Apr. 7, 2011.

For correspondence or reprints contact: François Rouzet, INSERM, U698, Cardiovascular Bioengineering, Bichat-Claude Bernard Hospital, 46 Rue H.

Huchard, 75877 Paris Cedex 18, France.

E-mail: francois.rouzet@bch.aphp.fr

Published online Aug. 17, 2011.

${ }^{*}$ Contributed equally to this work.

COPYRIGHT @ 2011 by the Society of Nuclear Medicine, Inc.
}

$\mathbf{P}$ -selectin is an adhesion molecule expressed at the surface of endothelial cells and platelets on activation. It mediates leukocyte rolling on activated endothelium ( $(1)$ and leukocyte trapping on platelet aggregates (2), through the interaction with its counterreceptor P-selectin glycoprotein ligand 1. In the cardiovascular field, P-selectin expression is involved in the pathophysiology of 2 settings of prominent clinical relevance: the renewal and growth of biologically active (at risk) arterial thrombus (2) and endothelial activation after an acute transient myocardial ischemic event referred to as ischemic memory (3). Activated platelets expose granular P-selectin on their membrane surface, and myocardial injury is associated with activation of endothelial cells resulting in Weibel-Palade bodies exocytosis and overexpression of adhesion molecules $(1,4)$, which persists after ischemia has resolved. Therefore, $\mathrm{P}$-selectin promotes platelet, endothelium, and leukocyte interactions, whatever the cardiovascular events, representing an important molecular target in acute and in chronic cardiovascular diseases.

Consequently, many efforts have been made to develop imaging agents targeting P-selectin (5-11). These agents were built either on anti-P-selectin antibodies or on synthetic mimics of sialyl Lewis $\mathrm{X}\left(\mathrm{SLe}^{\mathrm{X}}\right)$, which is the natural ligand of P-selectin $(12,13)$. Although effective, the cost of synthesis of these agents and the risk of immunogenicity of antibodies preclude a potential translation to clinical use.

Fucoidan, referring to a type of sulfated polysaccharide derived from brown seaweed, is a naturally occurring mimic of $\mathrm{SLe}^{\mathrm{X}}(14)$. Our group recently compared the interaction of P-selectin with 3 low-molecular-weight (LMW) sulfated polysaccharides (15). The fucoidan exhibited the highest affinity for P-selectin, 2 orders of magnitude greater than the others, as well as the lowest nonspecific binding. Moreover, its pharmacologic effect as an in vivo selectin inhibitor has been assessed in various models of ischemia-reperfusion. After transient myocardial ischemia, fucoidan administration was associated with a decrease of neutrophil infiltration and pla- 
telet deposition in reperfused myocardium and finally reduced the extent of necrosis $(16,17)$.

Because fucoidan is likely to overcome the main limitations of prior targeting agents of P-selectin, we propose radiolabeled fucoidan as a novel approach for P-selectin molecular imaging. Its ability to detect platelet-rich arterial thrombus and endothelial activation after an acute ischemic event is evaluated in vivo in experimental models.

\section{MATERIALS AND METHODS}

\section{Fucoidan Chemical Characterization and Labeling Procedure}

The LMW fucoidan was obtained from Algues et Mer Co. The chemical composition (w/w) of fucoidan was $45 \%$ L-fucose, $25 \%$ Dglucuronic acid, and 27\% (\%S, 8.4; SD, 2\%-6\%) sodium ester sulfate. Sulfur elemental analysis was performed by Service Central de Microanalyse du CNRS. The LMW fucoidan average number molecular weight and average weight molecular weight, obtained from size-exclusion chromatography, were 7,200 and 9,600 g/mol, respectively (polymolecularity, 1.33). Size-exclusion chromatography was performed on a TSK gel G3000 PWXL (Interchim SA) with an LC10Ai pump (Shimadzu) and phosphate-buffered saline, $\mathrm{LiNO}_{3} 150 \mathrm{mM}, \mathrm{pH} 7.4$, as the eluent, at a flow rate of $0.5 \mathrm{~mL} / \mathrm{min}$ at $25^{\circ} \mathrm{C}$. The multi-angle laser light scattering apparatus was the EOS (18 detectors) from Wyatt Technology filled with a K5 cell and a gallium arsenide laser $(\lambda, 690 \mathrm{~nm})$. The other online detector was a differential refractometer (RID $10 \mathrm{~A}$; Shimadzu). A differential index of refraction of $0.15 \mathrm{~mL} / \mathrm{g}$ was used for all samples. Data were processed using Astra 5.3.4.14 software (Zimm order, 1 , with angles between $52^{\circ}$ and $142^{\circ}$ ).

Fucoidan was labeled according to a classic reduction reaction of pertechnetate. Sodium pertechnetate $\left({ }^{99} \mathrm{TcO}_{4}{ }^{-}\right.$, about $740 \mathrm{MBq}$ in $<100 \mu \mathrm{L}$ of saline, freshly eluted), $4 \mu \mathrm{g}$ of stannous chloride (1 $\mu \mathrm{g} / \mu \mathrm{L}$; Sigma-Aldrich), and $2 \mu \mathrm{g}$ of potassium borohydride ( $1 \mu \mathrm{g} / \mu \mathrm{L}$; Sigma-Aldrich) were added to a vial containing $10 \mu \mathrm{g}$ of LMW fucoidan $(1 \mathrm{mg} / \mathrm{mL})$ and left to incubate for $1 \mathrm{~h}$ at room temperature. The quality control was performed with instant thinlayer chromatography developed in methyl ethyl ketone buffer. The radiochemical purity was always superior to $99 \%$.

\section{In Vitro Binding Assay}

Binding of radiolabeled fucoidan to platelets has been assessed in adhesion conditions, likely to provoke their activation. The method of quantification of radiotracer binding was derived from a rotating radioimmunoassay technology (LigandTracer; Ridgeview Instruments). Falcon culture plates were coated with fibrinogen $(1.5 \mathrm{~mL}$ at $20 \mu \mathrm{g} / \mathrm{mL}$ ) overnight at room temperature. Plates were then washed with assay buffer ( $N$-(2-hydroxyethyl)piperazine- $N{ }^{\prime}$ (2-ethanesulfonic acid) $[20 \mathrm{mM}], \mathrm{NaCl}[150 \mathrm{mM}]$, and $\mathrm{CaCl}_{2}[1$ $\mathrm{mM}], \mathrm{pH}=7.4$ ), blocked overnight with $3 \%$ bovine serum albumin in the same buffer, and washed again. Blood was sampled from healthy volunteers $(n=2)$ who had taken no medication during the 2 previous weeks. Platelets were isolated according to a procedure previously published (18). Washed platelets $(1.5 \mathrm{~mL}$ at $300 \mathrm{G} \mathrm{L}^{-1}$ ) were left to incubate for $1 \mathrm{~h}$ at room temperature on the plates. Then, the supernatant was removed and replaced by 1.5 $\mathrm{mL}$ of radiolabeled fucoidan solution $\left(1.510^{-8} \mathrm{M}, 7 \mathrm{MBq}\right.$ ) (composition buffer). Fibrinogen-coated plates without platelets were used as controls. $(n=2)$. After equilibrium was reached, decaycorrected counts were averaged over 5 min.

\section{Biodistribution Studies}

For blood clearance assessment, 4 male Wistar rats were anesthetized with urethan $(1.22 \mathrm{~g} / \mathrm{kg})$, and a catheter $(0.7-\mathrm{mm}$ diameter; Jelco Critikon) was inserted into the left carotid for sampling. Each animal received a single injection (penis vein) of $50 \mathrm{MBq}$ of ${ }^{99 \mathrm{~m}} \mathrm{Tc}-$ fucoidan in a $250-\mu \mathrm{L}$ volume. Blood samples $(250 \mu \mathrm{L})$ were taken at $5,15,30,45,60,90,120,180$, and $240 \mathrm{~min}$ after injection. Saline $(250 \mu \mathrm{L})$ was injected immediately after each sampling to maintain blood volume and to rinse the catheter. For each sample, 1 aliquot of $100 \mu \mathrm{L}$ was taken for well counting (Cobra II; Packard).

Biodistribution of ${ }^{99 \mathrm{~m}} \mathrm{Tc}$-fucoidan was assessed in 4 male Wistar rats injected with an activity of $50 \mathrm{MBq}$ (corresponding to $0.7 \mu \mathrm{g}$ of fucoidan). Animals were euthanized with pentobarbital overdose 120 min after intravenous injection, and liver, spleen, heart, lungs, brain, thyroid, kidneys, penis, testis, thyroid, a sample of skin (plus hairs), skeletal muscle, and colon were excised, washed, and weighed. Blood, urine, bone (femur), and bone marrow were also sampled. Each tissue and the injected solution were aliquoted for well counting. After correction for residual activity in the injection site and decay, results were expressed as the percentage of administered dose per gram of organ and per whole organ. Blood, muscles, bone, and skin (and hairs) were assumed to represent, respectively, $6 \%, 40 \%, 10 \%$, and $18 \%$ of the body weight (19).

\section{Experimental Models}

Two kinds of experimental models were selected to evaluate the ability of radiolabeled fucoidan to detect P-selectin expression: platelet-rich arterial thrombi and myocardial ischemia-reperfusion. These 2 settings were chosen because they were clinically relevant, and both were associated with an overexpression of platelet and endothelial P-selectin, respectively. The procedures and the animal care complied with the principles of animal care formulated by the National Society for Medical Research. This study was conducted under authorization of the French Direction of the Veterinary Services (no. 75-214).

Platelet-Rich Arterial Thrombi. Investigations were performed in 2 models because they involve platelet-leukocyte interactions at different degrees. These models allow characterization of the ${ }^{99 m}$ Tc-fucoidan uptake not only within the thrombus itself but also in the underlying tissues.

The first model was abdominal aortic aneurysms (AAAs) in the rat. The elastase model (20) was performed in 9 male Lewis rats, aged $12 \mathrm{wk}$, purchased from CEJ. The model was induced under general pentobarbital $(4 \mathrm{mg} / 100 \mathrm{~g}$ of body weight $)$ anesthesia by in situ intraluminal pressurized perfusion of pig pancreatic elastase ( 3 units, E1250 [Sigma], 1-h perfusion) within an isolated segment of the abdominal aorta. This model is characterized by a biologically active intraluminal thrombus $(21,22)$. Imaging was performed $15 \mathrm{~d}$ later. Three additional sham-operated rats served as controls.

The second model was infective endocarditis in the rat. Six male Wistar rats (Janvier) were used in the study. The procedure was derived from the classic model, as described previously (23). In brief, a polyethylene catheter (PE10; Clay Adams) was inserted into the left ventricle under pressure monitoring through the right carotid artery in anesthetized rats (ketamine-xylazine). The catheter remained indwelling throughout the experiment to induce a thrombotic vegetation formation. Twenty-four hours after catheterization, rats underwent bacterial inoculation $\left(10^{8}\right.$ colony-forming units of Enterococcus faecalis JH2-2) by the left jugular vein under halothane anesthesia. Vegetations of infective endocarditis are platelet-rich thrombi, in 
which biologic activity is enhanced by bacterial colonization (23). Imaging was performed $4 \mathrm{~d}$ later.

Myocardial Ischemia-Reperfusion. Nine male Wistar rats (Janvier) were used in the study. The proximal left anterior descending coronary artery was occluded using a suture around a catheter for $20 \mathrm{~min}$ and then was reperfused by cutting the suture along the catheter in rats under general anesthesia (ketamine-xylazine) and positive pressure ventilation (24). Injection of the radiotracer was performed after $2 \mathrm{~h}$ of reperfusion.

To evidence a nonspecific retention of radiolabeled compounds in the injured reperfused myocardium, a control study was performed in 2 additional rats. We used ${ }^{99 \mathrm{~m}} \mathrm{Tc}$-aprotinin, a glycoprotein with a molecular weight $(6.5 \mathrm{kDa})$ that is similar to that of fucoidan, with a radiolabeling process previously published $(25,26)$ as a control radiotracer.

\section{Scintigraphy}

All SPECT acquisitions were performed on a dedicated smallanimal $\gamma$-camera ( $\gamma$ IMAGER-S; Biospace Lab) equipped with parallel low-energy, high-resolution collimators, $(256 \times 256$ matrix), and a $15 \%$ energy window centered on $140 \mathrm{keV}$. In vivo scintigraphy was performed $2 \mathrm{~h}$ after intravenous injection of the radiotracer, during $45 \mathrm{~min}$, under anesthesia with intraperitoneal pentobarbital (4 mg/100 g of body weight; Ceva Santé Animale). Additionally, CT (micro-CT; Biospace Lab) was performed at the same time as scintigraphy for coregistration of the images. Iterative reconstruction of SPECT acquisitions was performed on a Xeleris 2 workstation (GE Healthcare) using ordered-subsets expectation maximization (4 iterations, 8 subsets) with Butterworth 3-dimensional postfiltering (cutoff frequency, 1.3 cycle/ pixel; order, 5). Reconstructed slices were visually assessed in 3 planes (axial, coronal, and sagittal) with and without CT coregistration to determine the presence of a focal uptake in the heart area or in the infrarenal aorta according to the model. Quantification was performed by calculating the ratio between the activity (mean counts) in the injured area and in a normal region (background) on short-axis slices. The background activity was derived from a region of interest drawn over the suprarenal aorta avoiding renal activity (AAA model) or blood pool in the right ventricle (infective endocarditis and ischemia-reperfusion models).

\section{Autoradiography}

After completion of SPECT, animals were euthanized with a pentobarbital overdose. Tissue samples were frozen and cut into transverse sections $(20-\mu \mathrm{m}$ thickness) that were exposed in a digital radioimager (Instant Imager; Packard) for $12 \mathrm{~h}$. Quantification was performed by calculating the ratio between the activity (mean counts $/ \mathrm{mm}^{2}$ corrected for background) of the relevant sample (heart or aorta) and the activity of a region of interest drawn on a reference tissue (normal myocardium remote from the vegetation or ischemic area, or thoracic aorta) $(21,23,24)$. In ischemiareperfusion studies, we estimated the cross-sectional extent of injured myocardium as the percentage of surface with increased radioactivity, compared with the surface of the left ventricular section on a slice perpendicular to the long axis of the heart taken in the midventricular segment.

\section{Histology and Immunohistochemistry}

At sacrifice, relevant tissue samples were fixed in paraformaldehyde for $24 \mathrm{~h}$, embedded in paraffin, and cut into $5-\mu \mathrm{m}$ sections for morphologic analysis. Serial sections were stained with Masson trichrome to visualize cells, nuclei, and fibrin and stained with
Alcian blue coupled with nuclear red to reveal areas of sulfated polysaccharide material and their relation to cell nuclei. After radioimager exposure, tissue sections used for autoradiography were also stained with Masson trichrome or Alcian blue coupled with nuclear red for coregistration. Immunohistochemistry studies were performed on 5 - $\mu \mathrm{m}$ cryostat sections using goat antimouse P-selectin antibody (SC 6943; Santa Cruz Biotechnology) (1/50) revealed by an antigoat antibody conjugated with horseradish peroxidase, followed by the 3,3-diaminobenzidine reaction.

\section{Statistical Analysis}

Continuous variables were expressed as median and range and compared by use of the Mann-Whitney $U$ test. Linear regression and Pearson correlation coefficients were performed in ischemiareperfusion $(n=9)$ to relate ${ }^{99 \mathrm{~m}} \mathrm{Tc}$-fucoidan activity ratios obtained in vivo by SPECT to the extent of injured myocardium measured by autoradiography. A $P$ value of less than 0.05 was considered significant.

\section{RESULTS}

\section{In Vitro Binding of ${ }^{99 m}$ Tc-Fucoidan to Platelets}

Binding of ${ }^{99 \mathrm{~m}} \mathrm{Tc}$-fucoidan to fibrinogen-coated plates was low with $776 \pm 14$ counts per second (cps) and $770 \pm 17 \mathrm{cps}$ (means $\pm \mathrm{SD}, n=2$ ) at equilibrium. In the same conditions, binding of ${ }^{99} \mathrm{~m}$ Tc-fucoidan was more than 10 -fold increased when platelets were allowed to adhere to the plate on the rotational device, reaching 9,867 $\pm 72 \mathrm{cps}$ and 8,401 \pm 42 cps, respectively, with platelets from 2 different donors.

\section{Biodistribution of ${ }^{99 m}$ Tc-Fucoidan}

After intravenous injection, ${ }^{99 \mathrm{~m}} \mathrm{Tc}$-fucoidan was cleared from the blood according to a 2-compartment model. The rapid component $(\alpha)$ accounted for $68 \%$ of the injected activity and had an effective half-life of $19 \mathrm{~min}$. The slow component $(\beta)$ accounted for $32 \%$ of the injected activity and had a much longer effective half-life of $351 \mathrm{~min}$. Thus, after a rapid decrease after injection, blood activity reached a plateau at a median value of $10.6 \%$ of the injected dose (8.9\%-12.5\%) after $120 \mathrm{~min}$ (Fig. 1). The tissue distribution of activity assessed $2 \mathrm{~h}$ after intravenous injection of ${ }^{99 \mathrm{~m}}$ Tc-fucoidan is presented in Table 1 and is illustrated in a normal rat (Supplemental Fig. 1; supplemental materials are available online only at http://jnm.snmjournals.org). Sequential whole-body acquisitions showed an early renal uptake of the tracer, followed by the bladder, with a high activity in urine, indicating a renal elimination of radiolabeled hydrophilic LMW fucoidan.

\section{Endocarditic Vegetations}

Visual analysis of SPECT/CT (Fig. 2) showed an uptake in the aortic valve area in all rats. Additional uptake foci were detectable in the left ventricular apex in some animals. The vegetation-to-background uptake ratio was 5.2 (2.7-

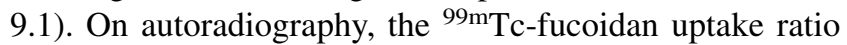
was 17 (14-32). Coregistration of autoradiography with histologic analysis performed after routine staining of tissue slices showed a clear delineation of the tracer uptake within the vegetations (Supplemental Fig. 2; Fig. 3). There was no increased tracer uptake above the background activity in 
Blood activity (\% injected dose, $n=4$ )

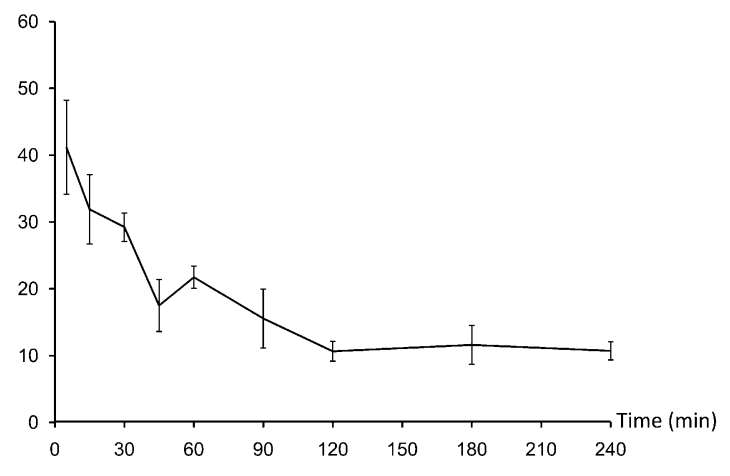

FIGURE 1. Blood clearance of $99 \mathrm{~m} T c$-fucoidan. After correction for residual activity in injection site and decay, results are expressed as percentage of administered dose remaining in blood pool according to time, assuming that blood represents $6 \%$ of body weight.

Alcian blue-positive injured myocardium or in edematous areas underlying vegetations. A similar pattern was found for P-selectin immunostaining on contiguous slices (Fig. 3).

\section{Aneurysmal Intraluminal Thrombus}

Macroscopic examination at sacrifice showed the presence of an aneurysm of the abdominal aorta in all animals that underwent an elastase infusion, coated with a mural thrombus on its luminal side. The size of aneurysm may vary among animals (median infrarenal aorta diameter, 3.3 $\mathrm{mm}$ [2.1-6.3] vs. $1.2 \mathrm{~mm}[1.1-1.3]$ in controls, $P=0.002)$.

Visual analysis of SPECT/CT (Supplemental Fig. 3) showed an uptake in the abdominal aorta area in all rats, with a median ${ }^{99 \mathrm{~m}} \mathrm{Tc}$-fucoidan uptake ratio of 3.6 (2.2-8.5), whereas no signal was detectable in sham-operated animals (uptake ratio, $1.1[0.9-1.1], P=0.01)$. In this model of aneurysmal mural thrombus, the radiotracer uptake ratio is

TABLE 1

Biodistribution of ${ }^{99 m}$ Tc-Fucoidan in Rats $(n=4)$ 2 Hours After Intravenous Injection

\begin{tabular}{lcc}
\hline Organ & $\begin{array}{c}\text { Percentage of } \\
\text { administered dose } \\
\text { per gram of tissue }\end{array}$ & $\begin{array}{c}\text { Percentage of } \\
\text { administered dose } \\
\text { per organ }\end{array}$ \\
\hline Blood & $0.624 \pm 0.042$ & $11.73 \pm 0.79$ \\
Kidneys & $4.801 \pm 0.681$ & $12.17 \pm 1.49$ \\
Urine & $34.171 \pm 11.95$ & $18.07 \pm 5.54$ \\
Liver & $0.619 \pm 0.082$ & $7.85 \pm 1.10$ \\
Spleen & $0.213 \pm 0.055$ & $0.182 \pm 0.029$ \\
Bones & $0.559 \pm 0.093$ & $16.58 \pm 2.73$ \\
Muscle & $0.040 \pm 0.012$ & $5.02 \pm 1.53$ \\
Skin & $0.164 \pm 0.075$ & $9.20 \pm 4.16$ \\
Heart & $0.110 \pm 0.009$ & $0.129 \pm 0.027$ \\
Lungs & $0.172 \pm 0.028$ & $0.270 \pm 0.047$ \\
Thyroid & $0.250 \pm 0.100$ & $0.005 \pm 0.002$ \\
Brain & $0.014 \pm 0.002$ & $0.019 \pm 0.001$ \\
\hline
\end{tabular}

*Because there was no urination during study, this is cumulative activity of first $2 \mathrm{~h}$ after injection.

Results are expressed as mean \pm SD.

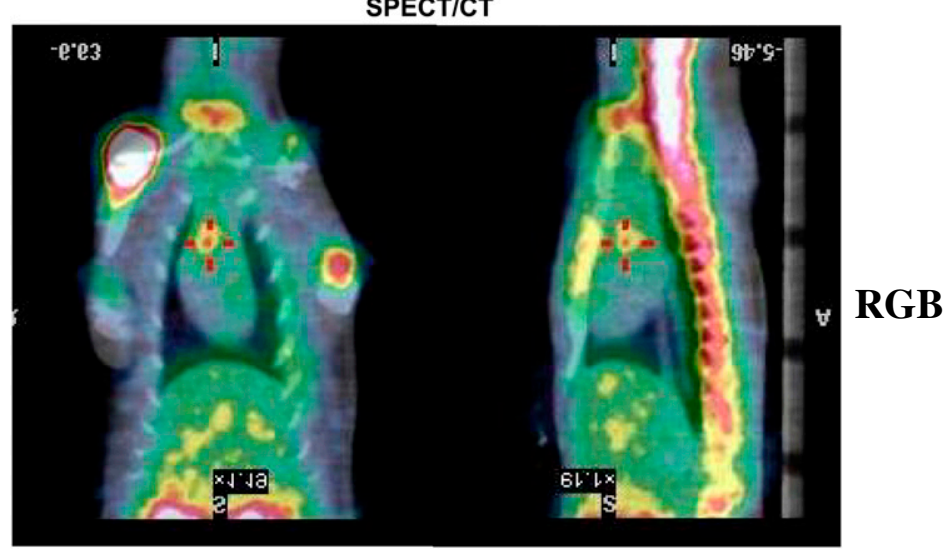

FIGURE 2. Aortic valve endocarditis in rat. Coronal and sagittal slices on SPECT/CT coregistration are shown, with an example of focal ${ }^{99 m}$ Tc-fucoidan uptake (red cross) in aortic valve area. Additional uptake is seen in spine and upper extremities of humeral bones.

lowered by the high background activity related to the renal elimination of the tracer (Supplemental Fig. 3). On auto-

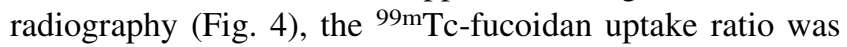
$9.1(6.3-15.8)$ in AAA and $1.0(1.0-1.2)$ in controls $(P=$ $0.01)$. Coregistration of autoradiography with histologic analysis performed after routine staining of tissue slices showed a clear delineation of the tracer uptake within the mural thrombi and a markedly lower uptake of the aorta wall (Fig. 4). Comparative analysis of autoradiography and P-selectin immunostaining showed the colocalization of 99mTc-fucoidan uptake and P-selectin (Fig. 4).

\section{Endothelial Activation in Ischemia-Reperfusion}

Two hours after reperfusion, a focal uptake of ${ }^{99 \mathrm{~m}} \mathrm{Tc}-$ fucoidan had been detected in all animals, with an uptake ratio of 4.1 (2.3-6.2) on SPECT (Fig. 5A). On autoradiography, the ${ }^{99 \mathrm{~m}} \mathrm{Tc}$-fucoidan uptake ratio was 24.9 (4.1-44.0). The uptake was enhanced at the epicardium and at the boundary between the area at risk and normal myocardium (Fig. 5B). P-selectin immunostaining was localized in the intramyocardial vasculature in the ischemia-injured area. There was a good agreement between ${ }^{99 \mathrm{~m}} \mathrm{Tc}$-fucoidan uptake ratio quantified in vivo by SPECT and the extent of its uptake on autoradiography (Fig. 5C), suggesting a reliable noninvasive estimation of the ischemia-injured area.

Two additional rats with ischemia-reperfusion were injected with ${ }^{99 \mathrm{~m}}$ Tc-aprotinin (Supplemental Fig. 4) to assess the nonspecific retention in the reperfused myocardium of the glycoprotein, which has a molecular weight similar to that of fucoidan (6.5 and $7.2 \mathrm{kDa}$, respectively). No focal uptake on SPECT or increased activity within the ischemiainjured area on autoradiography (uptake ratio was 1 for both SPECT and autoradiography; $P=0.03$, compared with ${ }^{99 \mathrm{~m}}$ Tc-fucoidan) was detectable.

\section{DISCUSSION}

This study shows the following. First, through P-selectin molecular imaging in 2 models of platelet-rich arterial thrombi, 


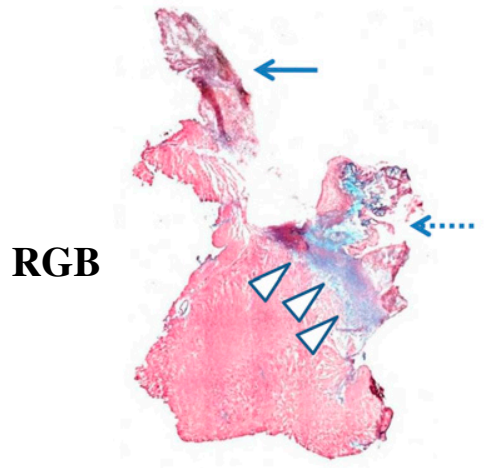

Alcian blue

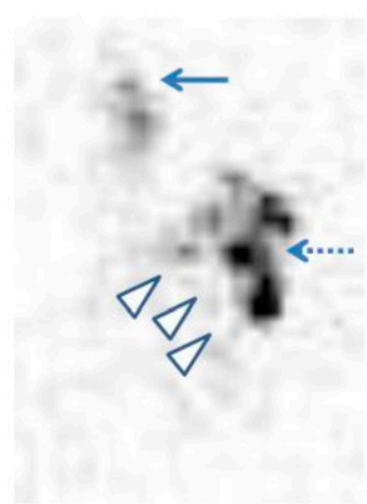

Autoradiography

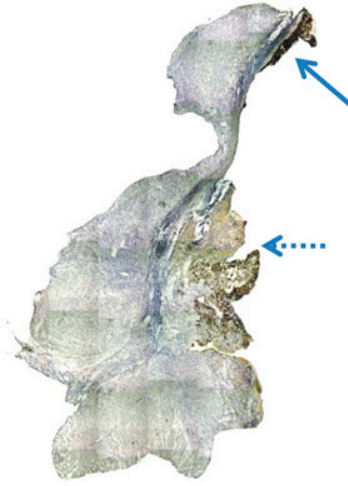

P-selectin immunostaining
FIGURE 3. Aortic valve endocarditis in rat. Comparison of histology (5- $\mu \mathrm{m}$ thickness, Alcian blue staining), autoradiography, and P-selectin immunostaining (with nuclear counterstaining) in same vegetation (adjacent slices for histology and autoradiography but not for immunostaining). Two vegetations are present on this sample: one attached to aorta wall damaged by catheter path (solid arrow) and second attached to aortic valve (dotted arrow). There is clear colocalization of $99 \mathrm{mTc}$-fucoidan uptake and immunostaining on both vegetations but not within damaged myocardium underlying vegetation (proteoglycans are stained in blue, indicated by arrowheads).
99mTc-fucoidan SPECT was able to detect the presence of thrombi with a good sensitivity. Second, in a model of myocardial ischemia-reperfusion, ${ }^{99 m} \mathrm{Tc}$-fucoidan SPECT was able to detect a persistent endothelial activation at $2 \mathrm{~h}$ after reperfusion, and the magnitude of the signal was correlated to the extent of myocardium that underwent transient ischemia. Third, there is an excellent sensitivity and selectivity at a tissue level of the uptake and retention of ${ }^{99 \mathrm{~m} T c-f u c o i d a n}$ in both arterial thrombus and ischemia-reperfusion models.

\section{P-Selectin-Targeted Imaging Agents}

The interaction between P-selectin and P-selectin glycoprotein ligand 1 involves the tetrasaccharide $\mathrm{SLe}^{\mathrm{X}}$ (27-29). Many studies have shown that mimics of SLe ${ }^{\mathrm{X}}$, oligosaccharides, and sulfated polysaccharides such as heparin, heparan sulfate, dextran sulfate, and fucoidan or some of their derivatives were able to interact with P-selectin $(12,13,30)$. Among them, fucoidans are a family of polysaccharides extracted mainly from brown algae and containing a substantial percentage of L-fucose and ester sulfate. Recently, we showed that LMW fucoidan prevented P-selectin binding to $\mathrm{SLe}^{\mathrm{X}}$ with a half-maximal inhibitory concentration of $20 \mathrm{nM}$ and exhibited a high affinity for immobilized P-selectin with a dissociation constant of $1.2 \mathrm{nM}$ together with a low binding to a nonspecific immunoglobulin. Moreover, the binding of fucoidan to human platelets increased with the level of platelet activation, and the binding of anti-P-selectin antibody to activated platelets was inhibited by fucoidan, demonstrating the specificity of the interaction between fucoidan and Pselectin (15). These data suggested the ability of fucoidan as a targeting vector for P-selectin.

Compared with agents used in previous studies to target P-selectin, such as antibodies or synthetic SLe ${ }^{\mathrm{X}}$, radiolabeled fucoidan presents some advantages. First, its toxicity has been investigated in recent years for the development of medicines, cosmetics, and food supplements, with no severe adverse effect reported in animals or humans (Material Safety Data, Algues \& Mer) (31,32). We have already tested a high dose of fucoidan $(5 \mathrm{mg} / \mathrm{kg} / \mathrm{d}$ intramuscularly during $2 \mathrm{wk}$ ) in experimental therapeutics in rats without detectable side effects (33). Furthermore, animal studies showed that through selectin inhibition, fucoidan decreased $\mathrm{T}$ lymphocyte activation and proliferation and eosinophil recruitment to sites of allergic inflammation (34). Because of the high sensitivity of nuclear imaging, the amount of labeled compound administered in the present study $(0.5-2$ $\mu \mathrm{g} / \mathrm{animal})$ was at least 3 orders of magnitude below that likely to induce a pharmacologic effect $(16,17)$. Second, fucoidan is widely available at low cost because of its extraction from algae, avoiding complex and expensive steps of production and purification required for antibodies or synthetic SLe ${ }^{X}$. This is critical for industrial development and further clinical spreading. Finally, all the steps from labeling to image visualization and interpretation are

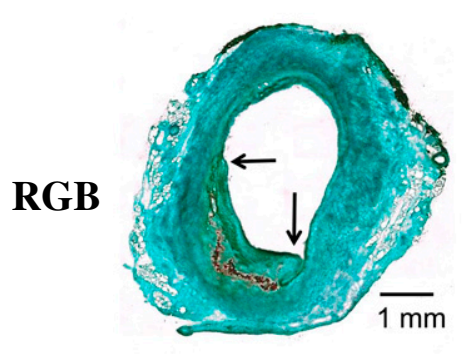

Masson's trichrome

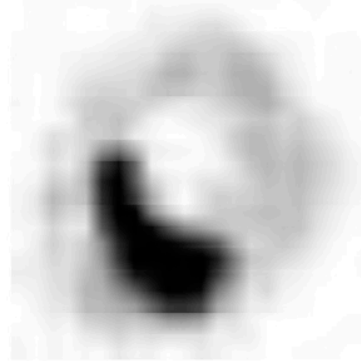

Autoradiography

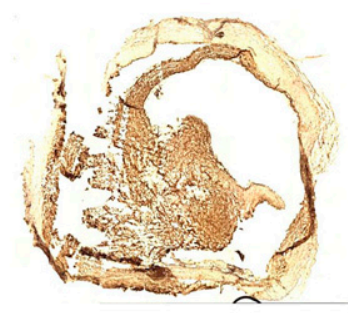

P-selectin immunostaining
FIGURE 4. Intraluminal thrombus in AAA in rat. Compared analysis of histology (Masson trichrome) and autoradiography on same slice (cryostat section, 20- $\mu \mathrm{m}$ thickness; Masson trichrome staining has been performed after autoradiographic acquisition) and P-selectin immunostaining with nuclear counterstaining (different sample). Mural thrombus on Masson trichrome staining (arrows) matched 99mTcfucoidan uptake on autoradiography. P-selectin positivity on immunostaining was localized within thrombus, which is spreading at surface of aneurysm wall. 
FIGURE 5. Ischemia-reperfusion after transient occlusion of left anterior descending coronary artery in rat. (A) SPECT/CT acquired after $2 \mathrm{~h}$ of reperfusion shows high ${ }^{99 \mathrm{mTC}}$ fucoidan uptake in anterior wall (arrow). 99mTc-fucoidan uptake on SPECT is quantified on short-axis slices as ratio between activity of region of interest drawn around focal ventricular uptake (line) and background activity (white dotted circle over right ventricular blood pool). (B) Autoradiography of axial slice of midventricular portion of heart: uptake in area at risk matched P-selectin immunostaining (peroxidase/3,3-diaminobenzidine; magnification, $\times 40$ ) on vessels wall. Conversely, no significant radiotracer uptake or immunostaining was detected in remote myocardium. (C) At left is cross-sectional extent of injured myocardium quantified on autoradiographic slice perpendicular to long axis of heart taken in midventricular segment, as percentage of surface of area with increased radioactivity (red line) to total surface of left ventricle (blue line). At right is correlation between ${ }^{99 m}$ Tc-fucoidan uptake on SPECT and cross-sectional extent of injured myocardium on autoradiography $(\mathrm{Y}=2.223+9.681 \times \mathrm{X} ; R=0.7 ; P=0.04)$.

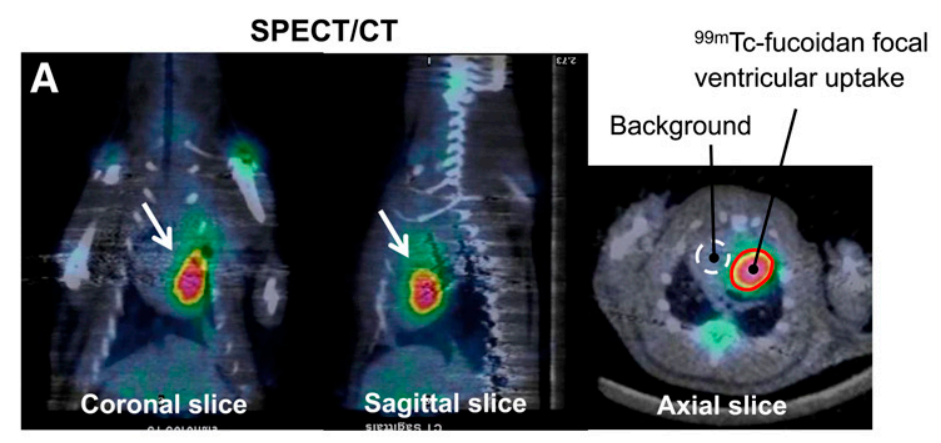

B

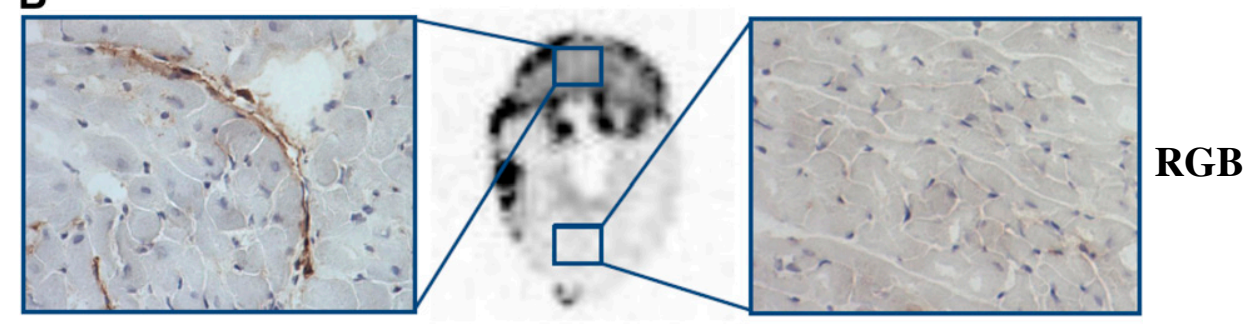

C
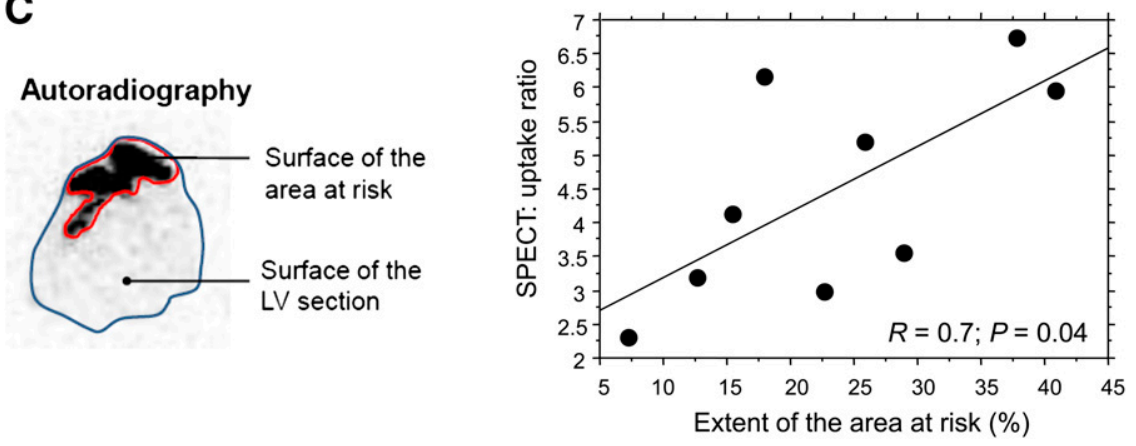

quite straightforward. Indeed, the radiolabeling procedure used in the present study is routinely performed in clinical practice, and the radiolabeling yield is acceptable for clinical use. However, because this is a preclinical proof-ofconcept study, the procedure does not comply with good manufacturing practice requirements, and additional work has to be done before human administration.

In the present study, the selectivity of radiolabeled fucoidan uptake and retention by tissues overexpressing P-selectin is supported by pieces of 2 evidence: in the endocarditis model, there was no significant activity in the myocardium underlying vegetations, although histologic analysis and previous studies (23) accounted for inflammation, cell apoptosis, and tissue degradation, likely to be associated with an increased diffusion of small molecules across the capillary membrane; and in the ischemia-reperfusion model, the administration of a polypeptidic radiotracer (aprotinin) of a molecular weight similar to that of fucoidan was not associated with significant activity in the area at risk. These data suggest that the presence of exposed P-selectin, either by platelets or by endothelium, is necessary for the retention of radiolabeled fucoidan at least at the time imaging has been performed.

\section{Comparison with Phosphatidylserine Targeting (Radiolabeled Annexin V)}

Besides P-selectin, activated platelets also express phosphatidylserine on the outer leaflet of their membrane, which is the hallmark of their procoagulant activity and a target for radiolabeled annexin (35). In our previous study investigating the ability of annexin $\mathrm{V}$ to detect the procoagulant activity of activated platelets in vegetations of infective endocarditis, we found a mild although significant uptake in the myocardium underlying vegetations, associated with terminal deoxynucleotidyl transferase-mediated dUTP nick-end labeling positivity (23). A similar pattern was observed on mural thrombi of AAA in rats (21). Because annexin binds to phosphatidylserine, which is expressed by both activated platelets and apoptotic cells, this uptake is probably related to cardiomyocyte apoptosis induced by protease release in the vicinity of the thrombus (36). In the present study, we found no significant activity in the myocardium underlying the vegetations with ${ }^{99 \mathrm{~m} T c-f u c o i-}$ dan. Thus, compared with radiolabeled annexin V, fucoidan uptake and retention are more localized within the thrombus (or vegetation) itself. From an imaging perspective, such an increased specificity may be a double-edged sword 
by decreasing the sensitivity of lesion detection. Indeed, even if vegetations and mural thrombi of AAA have been detected in all animals in the present study, and probably also because the expression of P-selectin is quantitatively lower than phosphatidylserine in platelet-rich thrombus, the uptake ratio on autoradiography was reduced by half, compared with radiolabeled annexin $\mathrm{V}(21,23)$.

Conversely, we found an opposite pattern in the setting of ischemia-reperfusion, with an uptake ratio twice (SPECT) and 3-fold (autoradiography) greater with radiolabeled fucoidan than annexin (24). In this setting, we can infer that the number of molecular targets is significantly different between annexin and fucoidan. Only some cardiomyocytes enter an apoptotic process, mainly located at the mid myocardium (37), without significant uptake of annexin by endothelium (38). To this regard, even if endothelial cells can also undergo apoptosis, this mechanism is prominent mainly during the first hour of reperfusion and is markedly decreased at $2 \mathrm{~h}$ (39). By contrast, activated endothelium represents a substantial volume of the area at risk, which is able to expose quickly large amounts of P-selectin from Weibel-Palade bodies during several hours after reperfusion (40).

This study shows that the intensity of signal detected in vivo by SPECT is correlated with the extent of myocardium that underwent an episode of transient ischemia. This finding is important for the retrospective diagnosis of recent ischemia in the absence of necrosis as well as from a prognostic standpoint, because the probability of occurrence of a cardiac event is related to the extent of the area at risk. Such a relationship should also provide the opportunity to assess noninvasively the potential cardioprotective effect of adjunctive therapies in reperfusion injury. Additionally, the biodistribution study shows a low uptake of ${ }^{99 \mathrm{~m}} \mathrm{Tc}$-fucoidan in the brain, suggesting the absence of diffusion of the radiolabeled compound across the blood-brain barrier in normal conditions. Therefore, radiolabeled fucoidan would potentially affect the diagnostic work-up in patients suspected of having undergone a transient ischemic event, either cardiac or neurologic, after its complete resolution. Early diagnosis of inflammatory diseases of the brain would also be affected because they are associated with $\mathrm{P}$-selectin expression $(5,8)$.

\section{Limitations}

Animal models of ischemia-reperfusion are devoid of underlying atherosclerosis, which is associated with a widespread overexpression of $\mathrm{P}$-selectin. A diffuse expression of the target in vessels is likely to reduce the contrast of ${ }^{99 m}$ Tc-fucoidan uptake, thus leading to an underestimation of the area at risk. The intensity of remote myocardial ischemia and the delay after reperfusion are also likely to play a role in the level of ${ }^{99 m}$ Tc-fucoidan uptake. Further studies should assess the detectability of activated endothelium in less severe ischemic events (coronary subocclusion, brief episodes of ischemia) and the time course of ${ }^{99 \mathrm{~m} T c-}$ fucoidan uptake after reperfusion. Also, blood clearance of radiolabeled fucoidan will probably be slowed in renal failure, with a direct impact on background activity in cardiovascular settings. This change in biodistribution requires further investigation to determine whether it may affect lesion detectability and to determine the optimal delay between injection and imaging in this condition.

Fucoidan may present some structural heterogeneities after extraction from seaweeds, such as molecular weight variations or various compositions in fucose, sulfate, and uronic acid from one batch to another. To solve this issue, the use of a contrast-based biospecific fucoidan simply requires the establishment of a structural characteristics list corresponding to an optimal action, similar to what has been done for heparin, a polysaccharide of animal origin.

\section{CONCLUSION}

This study supports ${ }^{99 \mathrm{~m}}$ Tc-fucoidan as a relevant highly sensitive imaging agent for in vivo detection of biologic activities associated with $\mathrm{P}$-selectin expression, such as platelet-rich thrombus and a transient ischemic event. With regard to translation to clinical use, because of the large panel of cardiovascular diseases, including atherothrombosis, in which P-selectin-dependent mechanisms are involved, fucoidan could be an efficient imaging agent in cardiovascular pathologies. Moreover, because of the low cost of its preparation and availability, fucoidan seems to overcome some of the main limitations of previously reported P-selectintargeted imaging agents.

\section{DISCLOSURE STATEMENT}

The costs of publication of this article were defrayed in part by the payment of page charges. Therefore, and solely to indicate this fact, this article is hereby marked "advertisement" in accordance with 18 USC section 1734.

\section{ACKNOWLEDGMENTS}

We wish to thank Professor Luc Picton for his help in the determination of the molecular weight of fucoidan, Francoise Chau for supplying us with bacteria strains, and Dr. Anne Gruaz-Guyon for helpful commentaries. This study was supported by INSERM and by a grant of the French Society of Cardiology and the French Federation of Cardiology. We thank the Conseil Général d'Ile de France and the Paris Department for financial support to ATHIM-Medicen IdF and the Agence nationale de la recherche for financial support to INFLAM project-ANR TECSAN. INSERM U698 is supported by the FAD European integrated project (http:// www.fighting-aneurysm.org/). No other potential conflict of interest relevant to this article was reported.

\section{REFERENCES}

1. Gamble JR, Skinner MP, Berndt MC, Vadas MA. Prevention of activated neutrophil adhesion to endothelium by soluble adhesion protein GMP140. Science. 1990;249:414-417.

2. Yokoyama S, Ikeda H, Haramaki N, Yasukawa H, Murohara T, Imaizumi T. Platelet P-selectin plays an important role in arterial thrombogenesis by form- 
ing large stable platelet-leukocyte aggregates. J Am Coll Cardiol. 2005;45:12801286.

3. Gourdin MJ, Bree B, De Kock M. The impact of ischaemia-reperfusion on the blood vessel. Eur J Anaesthesiol. 2009;26:537-547.

4. Eniola AO, Willcox PJ, Hammer DA. Interplay between rolling and firm adhesion elucidated with a cell-free system engineered with two distinct receptorligand pairs. Biophys J. 2003;85:2720-2731.

5. Barber PA, Foniok T, Kirk D, et al. MR molecular imaging of early endothelial activation in focal ischemia. Ann Neurol. 2004;56:116-120.

6. Villanueva FS, Lu E, Bowry S, et al. Myocardial ischemic memory imaging with molecular echocardiography. Circulation. 2007;115:345-352.

7. McAteer MA, Schneider JE, Ali ZA, et al. Magnetic resonance imaging of endothelial adhesion molecules in mouse atherosclerosis using dual-targeted microparticles of iron oxide. Arterioscler Thromb Vasc Biol. 2008;28:77-83.

8. van Kasteren SI, Campbell SJ, Serres S, Anthony DC, Sibson NR, Davis BG. Glyconanoparticles allow pre-symptomatic in vivo imaging of brain disease. Proc Natl Acad Sci USA. 2009;106:18-23.

9. Palabrica TM, Furie BC, Konstam MA, et al. Thrombus imaging in a primate model with antibodies specific for an external membrane protein of activated platelets. Proc Natl Acad Sci USA. 1989;86:1036-1040.

10. Licha K, Debus N, Emig-Vollmer S, et al. Optical molecular imaging of lymph nodes using a targeted vascular contrast agent. J Biomed Opt. 2005;10:41205.

11. Kaufmann BA, Lewis C, Xie A, Mirza-Mohd A, Lindner JR. Detection of recent myocardial ischaemia by molecular imaging of P-selectin with targeted contrast echocardiography. Eur Heart J. 2007;28:2011-2017.

12. Varki A. Selectin ligands. Proc Natl Acad Sci USA. 1994;91:7390-7397.

13. Shodai T, Suzuki J, Kudo S, et al. Inhibition of P-selectin-mediated cell adhesion by a sulfated derivative of sialic acid. Biochem Biophys Res Commun. 2003; 312:787-793.

14. Li B, Lu F, Wei X, Zhao R. Fucoidan: structure and bioactivity. Molecules. 2008;13:1671-1695.

15. Bachelet L, Bertholon I, Lavigne D, et al. Affinity of low molecular weight fucoidan for P-selectin triggers its binding to activated human platelets. Biochim Biophys Acta. 2009;1790:141-146.

16. Omata M, Matsui N, Inomata N, Ohno T. Protective effects of polysaccharide fucoidin on myocardial ischemia-reperfusion injury in rats. J Cardiovasc Pharmacol. 1997;30:717-724.

17. Barrabes JA, Garcia-Dorado D, Mirabet M, et al. Antagonism of selectin function attenuates microvascular platelet deposition and platelet-mediated myocardial injury after transient ischemia. J Am Coll Cardiol. 2005;45:293-299.

18. Boulaftali Y, Adam F, Venisse L, et al. Anticoagulant and antithrombotic properties of platelet protease nexin-1. Blood. 2010;115:97-106.

19. Farris EJGJ. The Rat in Laboratory Investigation. 2nd ed. London, U.K.: JB Lippincott Company; 1942.

20. Anidjar S, Salzmann JL, Gentric D, Lagneau P, Camilleri JP, Michel JB. Elastase-induced experimental aneurysms in rats. Circulation. 1990;82:973-981.

21. Sarda-Mantel L, Coutard M, Rouzet F, et al. ${ }^{99 \mathrm{~m}} \mathrm{Tc}$-annexin-V functional imaging of luminal thrombus activity in abdominal aortic aneurysms. Arterioscler Thromb Vasc Biol. 2006;26:2153-2159.

22. Coutard M, Touat Z, Houard X, Leclercq A, Michel JB. Thrombus versus wall biological activities in experimental aortic aneurysms. J Vasc Res. 2010;47:355-366.
23. Rouzet F, Dominguez Hernandez M, Hervatin F, et al. Technetium 99m-labeled annexin $\mathrm{V}$ scintigraphy of platelet activation in vegetations of experimental endocarditis. Circulation. 2008;117:781-789.

24. Sarda-Mantel L, Hervatin F, Michel JB, et al. Myocardial uptake of ${ }^{99 \mathrm{~m} T c-}$ annexin- $\mathrm{V}$ and ${ }^{111}$ In-antimyosin-antibodies after ischemia-reperfusion in rats. Eur J Nucl Med Mol Imaging. 2008;35:158-165.

25. Schaadt BK, Hendel HW, Gimsing P, Jonsson V, Pedersen H, Hesse B. ${ }^{99 m}$ Tcaprotinin scintigraphy in amyloidosis. J Nucl Med. 2003;44:177-183.

26. Houard X, Rouzet F, Touat Z, et al. Topology of the fibrinolytic system within the mural thrombus of human abdominal aortic aneurysms. J Pathol. 2007; 212:20-28.

27. Vestweber D, Blanks JE. Mechanisms that regulate the function of the selectins and their ligands. Physiol Rev. 1999;79:181-213.

28. Ramphal JY, Zheng ZL, Perez C, Walker LE, DeFrees SA, Gaeta FC. Structureactivity relationships of sialyl Lewis X-containing oligosaccharides. 1. Effect of modifications of the fucose moiety. J Med Chem. 1994;37:3459-3463.

29. Ramachandran V, Nollert MU, Qiu H, et al. Tyrosine replacement in P-selectin glycoprotein ligand-1 affects distinct kinetic and mechanical properties of bonds with P- and L-selectin. Proc Natl Acad Sci USA. 1999;96: 13771-13776.

30. Koenig A, Jain R, Vig R, Norgard-Sumnicht KE, Matta KL, Varki A. Selectin inhibition: synthesis and evaluation of novel sialylated, sulfated and fucosylated oligosaccharides, including the major capping group of GlyCAM-1. Glycobiology. 1997;7:79-93.

31. Chung HJ, Jeun J, Houng SJ, Jun HJ, Kweon DK, Lee SJ. Toxicological evaluation of fucoidan from Undaria pinnatifidain vitro and in vivo. Phytother Res. 2010;24:1078-1083.

32. Irhimeh MR, Fitton JH, Lowenthal RM. Fucoidan ingestion increases the expression of CXCR4 on human CD34+ cells. Exp Hematol. 2007;35:989-994.

33. Luyt CE, Lepailleur-Enouf D, Gaultier CJ, Valdenaire O, Steg G, Michel JB. Involvement of the endothelin system in experimental critical hind limb ischemia. Mol Med. 2000;6:947-956.

34. Teixeira MM, Hellewell PG. The effect of the selectin binding polysaccharide fucoidin on eosinophil recruitment in vivo. Br J Pharmacol. 1997;120:1059-1066.

35. Rouzet F, Sarda-Mantel L, Michel JB, Le Guludec D. Molecular imaging of platelet activation in thrombus. $J$ Nucl Cardiol. 2009;16:277-286.

36. Fontaine V, Jacob MP, Houard X, et al. Involvement of the mural thrombus as a site of protease release and activation in human aortic aneurysms. Am J Pathol. 2002;161:1701-1710.

37. Dumont EA, Hofstra L, van Heerde WL, et al. Cardiomyocyte death induced by myocardial ischemia and reperfusion: measurement with recombinant human annexin-V in a mouse model. Circulation. 2000;102:1564-1568.

38. Dumont EA, Reutelingsperger CP, Smits JF, et al. Real-time imaging of apoptotic cell-membrane changes at the single-cell level in the beating murine heart. Nat Med. 2001;7:1352-1355.

39. Scarabelli TM, Stephanou A, Pasini E, et al. Different signaling pathways induce apoptosis in endothelial cells and cardiac myocytes during ischemia/reperfusion injury. Circ Res. 2002;90:745-748.

40. Chukwuemeka AO, Brown KA, Venn GE, Chambers DJ. Changes in P-selectin expression on cardiac microvessels in blood-perfused rat hearts subjected to ischemia-reperfusion. Ann Thorac Surg. 2005;79:204-211. 